\title{
Evaluación del efecto de una intervención grupal para la prevención de trastornos de la conducta alimentaria en adolescentes. Estudio piloto
}

Evaluation of the Effect of a Group Intervention for the Prevention of Eating Disorders in Adolescents. Pilot Study

\author{
Susana Montero Hernández, Marta San José de León, Javier Marín Rodríguez, \\ Bárbara Alonso Castro, Nerea Román Rodríguez de Tudanca, Carlos Bernabeu Serrano
}

Enfermeros especialistas en Salud Mental. Facultad de Enfermería y Fisioterapia de Alcalá de Henares, Madrid.

Contacto: susy_89madrid@hotmail.com

Fecha de recepción: 30 de abril de 2018 / Fecha de aceptación: 7 de junio de 2018

\begin{abstract}
Resumen
Los trastornos de la conducta alimentaria (TCA) son problemas relevantes de salud mental que afectan principalmente a mujeres adolescentes y jóvenes. En su etiología convergen factores biológicos, psicológicos y sociales y, por lo tanto, el tratamiento debe considerar esta multicausalidad. La enfermera de salud mental tiene un papel fundamental mediante la actuación sobre los factores de riesgo y así prevenir la enfermedad.

La insatisfacción corporal constituye un importante factor de riesgo y de mantenimiento de las patologías alimentarias. Las influencias socioculturales (sobre todo los medios de comunicación, familia y compañeros) contribuyen al desarrollo de la insatisfacción y, en consecuencia, aumentan la probabilidad de trastornos de la alimentación.

El objetivo es demostrar la eficacia de intervenciones grupales para mejorar la imagen corporal, la autoestima, la alfabetización mediática y otros factores que influyen en comportamientos y actitudes relacionados con la alimentación y poder prevenir la aparición de trastornos de la conducta alimentaria en adolescentes de entre 13 y 15 años mediante un estudio pre/post.
\end{abstract}

Palabras clave: trastornos de alimentación, educación para la salud, adolescente, prevención primaria.

\begin{abstract}
Eating disorders (ED) are significant mental health problems that primarily affect teenagers and young women. Biological, psychological and social factors contribute to their etiology and treatment should consider this. The Mental Health nurse plays a key role in influencing risk factors and preventing the disease.

Body dissatisfaction constitutes an important factor in the risk and maintenance of eating pathologies. Sociocultural influences (most notably the media, family and peers) contribute to the development of body dissatisfaction, and consequently increase the probability of developing eating disorders.

The objective is to show the effectiveness of group interventions to improve body image, self-esteem, media literacy and other factors that influence eating behaviors and attitudes and to be able to prevent the occurrence of eating disorders in adolescents between 13 and 15 years old through a pre/post study.
\end{abstract}

Keywords: eating disorders, health education, adolescent, prevention primary.

\section{Introducción}

Los trastornos de la conducta alimentaria (TCA) no son una patología tan moderna como a veces pudiéramos pensar, no son un problema nuevo, lo novedoso es la virulencia con la que se han presentado en la socie- dad actual y el cambio de actitudes que ésta ha tenido en sus conceptos de salud y de ideal estético.

La anorexia nerviosa (AN) es una de las patologías psiquiátricas más graves, más difíciles de tratar y con una elevada tasa de mortalidad ${ }^{4}$. 
Durante la adolescencia temprana, las niñas están cada vez más expuestas a los mensajes que promueven la cosificación de las mujeres y la pérdida de voz. Esta socialización ocurre de manera simultánea con la transición adolescente temprana, que implica cambios, ajustes y transformaciones en múltiples dominios de la vida. Para las niñas, esto a menudo desemboca en un aumento de las presiones para ajustarse a estándares de belleza irreales, como el ideal de cuerpo delgado ${ }^{7}$.

Aunque no se conoce la existencia de una etiología específica en el desarrollo de los trastornos alimentarios, sí hay evidencias que sugieren fuertemente una interacción de factores biopsicosociales que los crea y mantiene ${ }^{5,10}$.

Otras circunstancias que nos encontramos son la falta de conciencia de enfermedad, la frecuente aparición de la misma en la etapa adolescente, así como las dificultades para la detección y el tratamiento precoz de esta patología.

Todo esto pone en evidencia la necesidad de intervenir, por una parte, con la intención de prevenir la aparición de los TCA, generando programas de prevención primaria que favorezcan la reducción de la vulnerabilidad de la población ante los posibles factores de riesgo. Y, por otra parte, intervenir con la intención de detectarlos precozmente de manera que podamos garantizar, en mayor medida, el éxito terapéutico, al disminuir el tiempo entre el diagnóstico y el inicio del tratamiento ${ }^{4,11}$.

Muchos estudios describen cuales son las características más adecuadas para la mayor efectividad de un programa de prevención: Interactivo (vs. didáctico), multisesión (vs. única sesión) e intervenciones breves con sesión de refuerzo. Se recomienda, siempre que sea posible, un mínimo de 3 meses de seguimiento.

En cuanto a la edad y sexo, no existe consenso. En algunos artículos se recomienda intervenir a partir de los 15 años, en contradicción con metaanálisis y otros artículos que han encontrado que los programas son más eficaces cuando los participantes tienen menos de 15 años, ya que están aún en desarrollo. La mayoría de los estudios coinciden en que estos programas tienen mayor efecto en mujeres, debido al aumento en estas dos últimas décadas de la prevalencia de TCA especialmente en mujeres, además de tener casi 10 veces más posibilidades de presentar anorexia nerviosa que los hombres $^{4,7,13-18}$.

\section{Marco conceptual}

Este proyecto se ha desarrollado desde la teoría de relaciones interpersonales de Hildegard E. Peplau, descrita en su libro Relaciones interpersonales en Enfermería: un marco de referencia conceptual para la enfermería psicodinámica, publicado originalmente en 1952. Este marco teórico sugiere que los fenómenos interactivos que se producen en las relaciones enfermera-paciente tienen un impacto cualitativo en la evolución de los enfermos.

Hildegard E. Peplau se considera la madre de la Enfermería psiquiátrica. El modelo de Peplau es un modelo caracterizado en la atención centrada en el paciente. Cada encuentro enfermera-paciente, orientado por este modelo teórico, es una oportunidad transformadora y dignificante por su especial contenido humanístico. Esta humanización de los cuidados es la que siempre debe estar presente en el desarrollo de la enfermería de salud mental ${ }^{1-3}$.

\section{Justificación}

La alta prevalencia de trastornos alimenticios y conductas alimentarias desordenadas, sus consecuencias graves para la salud a corto y largo plazo, el alto coste del tratamiento, así como el éxito parcial del tratamiento, justifican el aumento de la atención política, social y sanitaria en la prevención primaria de trastornos de la alimentación.

Los principales objetivos de los programas escolares de prevención de trastornos alimentarios son la identificación y la crítica del modelo de belleza estética femenina, desarrollo del pensamiento crítico, es decir, descodificar mensajes de los medios sobre el cuerpo ideal, mensajes desafiantes, la glorificación de la delgadez..., motivar el desarrollo de un cuerpo sano y la auto-imagen, el aumento de la autoconfianza y autonomía con los compañeros, mejorando la comunicación y conocer habilidades para aprender a utilizar los medios de comunicación con eficacia y poder identificar los verdaderos mensajes de imágenes de cuerpo sano $^{19}$.

Para muchas adolescentes, no existe autoestima sin delgadez. El adelgazar se ha convertido en metáfora del éxito y el engordar del fracaso. La delgadez extrema, la belleza, es esencial para que una mujer triunfe, sea aceptable y exitosa en nuestra sociedad. Estos mensajes culturales los transmiten al parecer la fami- 
lia, los iguales y la prensa, los medios de comunicación de masas, que amplifican los valores socioculturales predominantes.

El culto al cuerpo y el canon estético de la delgadez esquelética, imposible para el común de las personas porque la osamenta no puede reducirse, obliga a inhumanos sacrificios 5 .

Por todo esto, y tras la revisión de los estudios más recientes, se considera muy importante la labor de prevención por parte de la enfermera de salud mental y se ha llevado a cabo este proyecto.

\section{Epidemiología}

Los TCA afectan principalmente a adolescentes y mujeres jóvenes y los estudios muestran que su prevalencia ha aumentado progresivamente desde la década de 1950 en adelante. Son la tercera enfermedad crónica más prevalente en la adolescencia después de la obesidad y el asma ${ }^{16}$.

Investigaciones realizadas en el extranjero han estimado que la prevalencia entre las jóvenes de AN oscila entre $0.5 \%$ y $1 \%$ y de Bulimia Nerviosa (BN) entre $1-2 \%$ y $4 \%$. Los TCA más frecuentes son no especificados (TANE), que alcanzan una prevalencia de hasta $14 \%$ según la definición utilizada.

El perfil de quienes presentan TCA ha ido cambiando gradualmente. Es así como hoy en día no solo afectan a las adolescentes de clase alta y excelente rendimiento académico, sino que además se presentan en jóvenes de sexo masculino, de diverso nivel socioeconómico, distintas etnias, individuos cada vez menores, y de diferente peso, tamaño y forma corporal.

También se ha demostrado que la ideación suicida es común en las adolescentes con dicho trastorno. Los intentos de suicidio se dan en un $22 \%$ de las pacientes con anorexia y un $11 \%$ en las pacientes con bulimia.

La insatisfacción corporal durante la adolescencia es común, pero no benigna. Entre el $17 \%$ y el $33 \%$ de los adolescentes informan insatisfacción corporal, con la cifra más alta para las niñas que los niños. La insatisfacción corporal es un importante problema de salud pública debido a su asociación con una serie de resultados negativos, que van desde la depresión y trastornos de la alimentación, con el uso de la cirugía estética, la obesidad y la pérdida de peso poco saludable, compor- tamientos superiores e inferiores a ejercer, como el tabaquismo. Como tal, la insatisfacción corporal ha sido el foco de la política del Gobierno en varios paí$\operatorname{ses}^{14}$.

\section{Metodología}

\section{Hipótesis}

Una intervención enfermera grupal basada en cuatro talleres sobre imagen corporal, alfabetización mediática, autoestima y la presión sociocultural junto con ciertas habilidades sociales en las alumnas de $4^{\circ}$ de Educación Secundaria Obligatoria (ESO) disminuye los factores de riesgo de los trastornos de conducta alimentaria.

\section{Objetivos}

\section{Objetivos generales:}

- Evaluar el efecto de una intervención grupal para la prevención de TCA en adolescentes de entre 13 y 15 años.

- Poner a prueba los métodos y procedimientos del estudio para conseguir un protocolo que garantice que se podrá responder a la pregunta de investigación.

\section{Objetivos especificos:}

- Analizar la evolución y situación actual de los trastornos de la conducta alimentaria: anorexia y bulimia nerviosa.

- Identificar las diferencias en la influencia ejercida por el ideal estético en función de la edad.

- Valorar la correlación entre la influencia ejercida por el ideal estético y la repetición de curso.

- Identificar las diferencias en la autoestima corporal en función de la edad.

- Valorar la correlación entre la autoestima corporal y la repetición de curso.

- Evaluar el efecto de la intervención diseñada sobre la influencia ejercida por el ideal estético.

- Evaluar el efecto de la intervención diseñada en la autoestima corporal.

\section{Diseño del estudio}

Estudio piloto de intervención sin grupo control: antesdespués. El programa es multisesión e interactivo. 


\begin{tabular}{|c|c|c|}
\hline SESIONES & CONTENIDOS & ACTIVIDADES \\
\hline SES IÓN 1 & $\begin{array}{l}\text { Presentación } \\
\text { Pretest } \\
\text { Dinámica de grupo } \\
\text { Imagen corporal } \\
\text { Vídeo } \\
\text { Discusión } \\
\text { Cierre }\end{array}$ & $\begin{array}{l}\text { Presentación } \\
\text { Normas del grupo: } \\
\text { confidencialidad, respeto, puntualidad... } \\
\text { Pretest } \\
\text { Dinámica: ¿Qué valoro en los demás? } \\
\text { Presentación PowerPoint: } \\
\text { ¿Qué es la imagen corporal? } \\
\text { Desarrollar una relación saludable con el cuerpo } \\
\text { (pubertad y desarrollo caracteres sexuales) } \\
\text { Importancia relativa del aspecto físico } \\
\text { Vídeo } \\
\text { Discusión } \\
\text { Cierre }\end{array}$ \\
\hline SES IÓN 2 & $\begin{array}{l}\text { Recapitulación } \\
\text { Crítica del modelo estético/publicidad } \\
\text { Dinámica de grupo } \\
\text { Vídeos } \\
\text { Discusión } \\
\text { Cierre }\end{array}$ & $\begin{array}{l}\text { Recapitulación: interviene en la imagen corporal } \\
\text { la comparación con los demás. } \\
\text { ¿Qué nos cuentan los anuncios sobre las mujeres? } \\
\text { Dinámica: Análisis fotos de modelos con y sin } \\
\text { retoques/análisis cambio de portada femenina a masculina } \\
\text { con las mismas frases. } \\
\text { Modelo estético en otras culturas y épocas. } \\
\text { Publicidad y negocio de adelgazamiento/rejuvenecimiento } \\
\text { Vídeos } \\
\text { Discusión } \\
\text { Cierre }\end{array}$ \\
\hline SES IÓN 3 & $\begin{array}{l}\text { Recapitulación } \\
\text { Autoimagen/Autoestima } \\
\text { Pensamientos negativos } \\
\text { Dinámica de grupo } \\
\text { Discusión } \\
\text { Cierre }\end{array}$ & $\begin{array}{l}\text { Recapitulación } \\
\text { ¿Qué es la autoestima? ¿Y la autoimagen? } \\
\text { Identificar pensamientos negativos/Importancia } \\
\text { pensamientos positivos } \\
\text { Dinámica: “Árbol de mi autoestima" } \\
\text { Dinámica de grupos: "Caricias interp ersonales" } \\
\text { Discusión } \\
\text { Cierre }\end{array}$ \\
\hline SES IÓN 4 & $\begin{array}{l}\text { Recapitulación } \\
\text { Pertenencia grupo social/influencia } \\
\text { amistades/asertividad } \\
\text { Dinámica de grupo } \\
\text { Vídeo } \\
\text { Discusión } \\
\text { Postest } \\
\text { Cierre }\end{array}$ & $\begin{array}{l}\text { Recapitulación } \\
\text { Relación autoestima con la influencia del grupo social. } \\
\text { Presión sociocultural. } \\
\text { Vídeo } \\
\text { De la infancia a la adolescencia. } \\
\text { Construcción de la identidad. } \\
\text { Vídeo } \\
\text { Asertividad. Estilos de comunicación. } \\
\text { Dinámica: "Ensayar la comunicación asertiva" } \\
\text { Vídeo con conclusiones. } \\
\text { Discusión } \\
\text { Postest } \\
\text { Cierre }\end{array}$ \\
\hline
\end{tabular}




\section{Procedimiento}

Una vez seleccionada la muestra para la intervención y habiendo recibido la aprobación por parte del IES seleccionado, se procede a contactar con los padres de las alumnas para proporcionarles la documentación informativa y el consentimiento informado.

La orientadora del colegio es la encargada de ponerse en contacto con los padres y las adolescentes para proporcionarles los documentos.

Se acude al colegio para comprobar que el aula reúne las condiciones necesarias para llevar a cabo la intervención correctamente y se recogen los consentimientos informados firmados tanto por los padres como por las adolescentes.

La intervención se ha desarrollado en un aula proporcionada por el IES para la misma, para influir lo menos posible en el horario académico de las alumnas y por tratarse de un lugar de referencia para ellas.

Se realizan 4 talleres:

- La primera sesión duró 60 minutos y se trabajó como tema principal la imagen corporal.

- La segunda sesión duró 60 minutos y se trabajó como tema principal la alfabetización mediática.

- La tercera sesión duró 60 minutos y se trabajó como tema principal la autoestima.

- La cuarta sesión duró 60 minutos y se trabajó como tema principal la presión sociocultural ${ }^{10}$.

Se imparte una sesión a la semana de 10:45 h a 11:45 h los viernes del mes de noviembre, por lo que la intervención grupal duró un mes.

Se pasaron los cuestionarios elegidos antes de la primera sesión y después de la última.

\section{Población y muestra}

Población diana: Adolescentes entre 13 y 15 años. Población a estudio: Mujeres entre 13 y 15 años cursando estudios de segundo ciclo de ESO en institutos de educación secundaria (IES) en Alcalá de Henares. Muestra: Para este pilotaje se ha utilizado una muestra de 10 sujetos estudiantes en uno de los IES de Alcalá de Henares (Madrid), para garantizar el óptimo funcionamiento de las dinámicas grupales necesarias para el correcto desarrollo de la intervención realizada.

\section{Criterios de inclusión y de exclusión}

Criterios de inclusión:

- Ser mujer.

- Tener entre 13 y 15 años.

- Estar cursando $4^{\circ}$ de ESO en el año escolar 20172018.

- Aceptar participar en el estudio y firma del consentimiento informado por representación.

\section{Criterios de exclusión:}

- Presentar o haber presentado diagnóstico de trastorno de conducta alimentaria.

- El no manejo o la no comprensión del idioma castellano.

- La incapacidad para llevar a cabo las actividades de la intervención diseñada.

\section{Tipo de muestreo}

Se ha realizado un muestreo tipo consecutivo no probabilístico.

\section{Variables a estudio}

Variables independientes:

- Edad: variable cuantitativa continua.

- Sexo: variable cualitativa dicotómica.

- Repetir curso: variable cualitativa dicotómica.

\section{Variables dependientes:}

- Influencia del cuerpo estético ideal medida con el cuestionario CIMEC: variable cuantitativa continua.

- Autoestima corporal medida con la escala BES: variable cuantitativa continua.

\section{Instrumentos de evaluación}

Cuestionario CIMEC sobre las influencias del cuerpo estético ideal:

CIMEC - Cuestionario de Influencias del Modelo Estético Corporal fue diseñado para intentar medir las influencias culturales relevantes que contribuyen a provocar, facilitar o justificar el adelgazamiento, especialmente por razones estéticas y sociales. Está compuesto por 40 ítems directos que evalúan la ansie- 
dad por la imagen corporal, la influencia de los modelos sociales y la influencia de las situaciones sociales. Las respuestas son evaluadas en una escala de 0 a 3 puntos. Una puntuación mayor denota una mayor influencia de los modelos sociales. Su puntuación máxima es de 80 , la mínima de 0 y el punto de corte es de $23 / 24$ puntos. Fue elaborado y validado en una muestra de jóvenes españolas (59 AN y 59 controles), igualadas en cuanto a edad y clase social. El cuestionario mostró una adecuada consistencia interna, así como una apropiada sensibilidad $(81,4 \%)$ y especificidad $(55,9 \%)$, por lo que podría servir como instrumento de cribado debido a la relación de la AN con las influencias socioculturales ${ }^{19,26,27}$.

Cuestionario BES, Body-Esteem Scale (Escala de autoestima corporal).

La Escala de Estima Corporal mide la satisfacción/insatisfacción corporal. Consta de 35 ítems que evalúan el tipo de emociones que le producen a la persona diferentes partes y características corporales, los cuales se valoran en una escala Likert de 5 puntos (1: «fuertes sentimientos negativos»; 2 : «sentimientos negativos moderados»; 3: «neutro»; 4: «sentimientos positivos moderados» y 5 : «fuertes sentimientos positivos»). Los factores de la escala son, para varones, «atractivo físico», «resistencia corporal» $\mathrm{y}$ «condición física general», y para mujeres, «atractivo sexual», «preocupación por el peso» y «condición física». La escala presenta buena fiabilidad en hombres $(0,78$ $0,87)$ y en mujeres $(0,81-0,86) 27-29$.

\section{Consideraciones éticas y legales}

A las participantes y a sus representantes se les informó verbalmente sobre el estudio y se les entregó un documento informativo con las características del estudio, solicitando su participación voluntaria.

La investigadora asegura la confidencialidad de la información en todas las fases del estudio. Los cuestionarios se guardarán en un sitio seguro, teniendo sólo acceso a ellos la investigadora.

El estudio tiene en cuenta los principios éticos básicos y los enunciados en la Declaración de Helsinki (revisión de Fortaleza 2012) y en el Convenio para la protección de los derechos humanos y la dignidad del ser humano con respecto a las aplicaciones de la biología y la medicina (Oviedo,1997). También se contempla la legislación sobre protección de datos (Ley Orgá- nica 15/1999, de 13 de diciembre, de Protección de Datos de Carácter Personal), la Ley 41/2002, de 14 de noviembre, Básica reguladora de la autonomía del paciente y de derechos y obligaciones en materia de información documentación clínica y la Ley 14/2007, de 3 de julio, de Investigación biomédica.

Se solicitó la autorización al IES seleccionado para la realización de la intervención.

El estudio se sometió al dictamen del Comité Ético de Investigación de la Universidad de Alcalá de Henares, resultando el informe favorable.

\section{Limitaciones del estudio}

Al tratarse de un estudio que carece de grupo control no se puede asegurar que los resultados obtenidos se deban exclusivamente a la intervención, ya que pueden haber influido otros factores no controlados.

\section{Recursos materiales y personales}

Recursos personales:

- Enfermera residente de salud mental.

- Director del IES, tutora y orientadora de las estudiantes que han participado en el proyecto.

\section{Recursos materiales:}

- Aula habilitada.

- Material fungible (folios, bolígrafos, pizarra, tizas...).

- Ordenador.

- Proyector.

- Fichas de las sesiones.

\section{Resultados}

Los 10 sujetos de la muestra seleccionada completaron la intervención en su totalidad. Todos los sujetos de investigación de este pilotaje son mujeres y estudian en el $4^{\circ}$ curso de la ESO. Al presentar esta común característica no se tendrá en cuenta para el análisis de resultados.

Dos sujetos de la muestra tienen 14 años y los ocho restantes tienen una edad de 15 años. Un solo sujeto de la muestra ha repetido curso. 
La distribución de frecuencias de la edad de los sujetos resulta según la tabla siguiente:

Edad

\begin{tabular}{|r|r|r|r|r|}
\hline & Frecuencia & Porcentaje & $\begin{array}{c}\text { Porcentaje } \\
\text { válido }\end{array}$ & $\begin{array}{c}\text { Porcentaje } \\
\text { acumulado }\end{array}$ \\
\hline Válido 14 & 2 & 20 & 20 & 20 \\
15 & 8 & 80 & 80 & 100 \\
Total & 10 & 100 & 100 & \\
\hline
\end{tabular}

Tabla 2

La variable repetición o no de curso distribuye su frecuencia según la tabla siguiente:

Repetidora

\begin{tabular}{|c|c|c|c|c|}
\hline & Frecuencia & Porcentaje & $\begin{array}{c}\text { Porcentaje } \\
\text { válido }\end{array}$ & $\begin{array}{l}\text { Porcentaje } \\
\text { acumulado }\end{array}$ \\
\hline Válido Sí & 1 & 10 & 10 & 10 \\
\hline No & 9 & 90 & 90 & 100 \\
\hline Total & 10 & 100 & 100 & \\
\hline
\end{tabular}

Tabla 3

Resumen de contrastes de hipótesis

\begin{tabular}{|c|c|c|c|c|}
\hline & Hipótesis nula & Prueba & Sig. & Decisión \\
\hline 1 & $\begin{array}{l}\text { Las categorías definidas por } \\
\text { Repetidora }=\text { No y Sí se producen } \\
\text { con probabilidades } 0,5 \text { y } 0,5\end{array}$ & $\begin{array}{l}\text { Prueba } \\
\text { binomial para } \\
\text { una muestra }\end{array}$ &, $021^{1}$ & $\begin{array}{l}\text { Rechace la } \\
\text { hipótesis } \\
\text { nula }\end{array}$ \\
\hline 2 & $\begin{array}{l}\text { La distribución de Edad es normal } \\
\text { con la media } 14,800 \\
\text { y la desviación estándar } 0,42\end{array}$ & $\begin{array}{l}\text { Prueba de } \\
\text { Kolmogorov- } \\
\text { Smirnov para } \\
\text { una muestra }\end{array}$ &, $000^{2}$ & $\begin{array}{l}\text { Rechace la } \\
\text { hipótesis } \\
\text { nula }\end{array}$ \\
\hline 3 & $\begin{array}{l}\text { La distribución de preBES es normal } \\
\text { con la media } 108,900 \\
\text { y la desviación estándar } 11,75\end{array}$ & $\begin{array}{l}\text { Prueba de } \\
\text { Kolmogorov- } \\
\text { Smirnov para } \\
\text { una muestra }\end{array}$ &, $200^{2.3}$ & $\begin{array}{l}\text { Conserve la } \\
\text { hipótesis } \\
\text { nula }\end{array}$ \\
\hline 4 & $\begin{array}{l}\text { La distribución de posBES es normal } \\
\text { con la media } 115,600 \\
\text { y la desviación estándar } 15,12\end{array}$ & $\begin{array}{l}\text { Prueba de } \\
\text { Kolmogorov- } \\
\text { Smirnov para } \\
\text { una muestra }\end{array}$ &, $200^{2.3}$ & $\begin{array}{l}\text { Conserve la } \\
\text { hipótesis } \\
\text { nula }\end{array}$ \\
\hline 5 & $\begin{array}{l}\text { La distribución de preCIMEC es normal } \\
\text { con la media } 25,700 \\
\text { y la desviación estándar } 13,14\end{array}$ & $\begin{array}{l}\text { Prueba de } \\
\text { Kolmogorov- } \\
\text { Smirnov para } \\
\text { una muestra }\end{array}$ &, $200^{2.3}$ & $\begin{array}{l}\text { Conserve la } \\
\text { hipótesis } \\
\text { nula }\end{array}$ \\
\hline 6 & $\begin{array}{l}\text { La distribución de posCIMEC es normal } \\
\text { con la media } 19,900 \\
\text { y la desviación estándar } 9,02\end{array}$ & $\begin{array}{l}\text { Prueba de } \\
\text { Kolmogorov- } \\
\text { Smirnov para } \\
\text { una muestra }\end{array}$ &, $200^{2.3}$ & $\begin{array}{l}\text { Conserve la } \\
\text { hipótesis } \\
\text { nula }\end{array}$ \\
\hline
\end{tabular}

Tabla 4 
Al tratarse de un pilotaje, y dado el tamaño muestral escogido para llevar a cabo la investigación, contamos con cierta limitación en la significación de los resultados, como se verá sucesivamente en este análisis.

Al no poderse garantizar que la muestra presente una distribución normal, por el escaso tamaño escogido para este pilotaje, se procede al uso del estadístico Kolmogorov-Smirnov como prueba no paramétrica.
Tras esta operación, se procede a la comparación de medias mediante análisis por t de Student en las variables que presentan una distribución normal.

En términos generales, sin atender a la subdivisión de la muestra para el análisis en función de las variables sociodemográficas, encontramos una mejoría en el resultado de ambos cuestionarios.

Estadís ticas de muestra única

\begin{tabular}{|l|r|r|r|r|}
\hline & N & \multicolumn{1}{|c|}{ Media } & \multicolumn{1}{c|}{$\begin{array}{c}\text { Des viación } \\
\text { es tándar }\end{array}$} & $\begin{array}{c}\text { Media de } \\
\text { error es tándar }\end{array}$ \\
\hline preBES & 10 & 108,9 & 11,752 & 3,716 \\
pos BES & 10 & 115,6 & 15,123 & 4,782 \\
preCIMEC & 10 & 25,7 & 13,141 & 4,155 \\
pos CIMEC & 10 & 19,9 & 9,024 & 2,854 \\
\hline
\end{tabular}

Tabla 5

Prueba de muestra única

\begin{tabular}{|c|c|c|c|c|c|c|}
\hline & \multicolumn{6}{|c|}{ Valor de prueba $=0$} \\
\hline & \multirow[t]{2}{*}{$\mathbf{t}$} & \multirow[t]{2}{*}{ gl } & \multirow{2}{*}{$\begin{array}{c}\text { Sig. } \\
\text { (bilateral) }\end{array}$} & \multirow{2}{*}{$\begin{array}{l}\text { Diferencia } \\
\text { de medias }\end{array}$} & \multicolumn{2}{|c|}{$\begin{array}{l}95 \% \text { de intervalo de } \\
\text { confianza de la diferencia }\end{array}$} \\
\hline & & & & & Inferior & Superior \\
\hline preBES & 29,304 & & 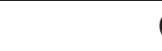 & 108,9 & 100,49 & 117,31 \\
\hline pos BES & 24,172 & & ( & 115,6 & 104,78 & 126,42 \\
\hline preCIMEC & 6,185 & & 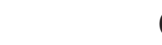 & 25,7 & 16,3 & 35,1 \\
\hline pos CIMEC & 6,974 & & 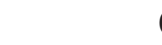 & 19,9 & 13,44 & 26,36 \\
\hline
\end{tabular}

Tabla 6

Se aprecia que la mejoría en el resultado de ambos cuestionarios es estadísticamente significativa $(p=0,000)$, no pudiendo atribuirse al azar la mejoría producida tras la aplicación de la intervención diseñada.

\section{Discusión}

Este estudio demuestra que con la intervención realizada se han producido efectos estadísticamente significativos en la mejora de la autoestima corporal y en la influencia ejercida por el ideal estético.

Los resultados son congruentes con varios estudios realizados con anterioridad. Diedrichs et al. llevaron a cabo en 2015 la intervención "Dove Confident Me: Single Session”, enfocada en imagen corporal, internalización del ideal estético y presión sociocultural. Se lleva a cabo una única sesión de 90 min en niñas y niños, pero se demuestra una mejora significativa en la autoestima corporal sólo en las niñas. El resto de factores de riesgo no tienen mejoras significativas. Señalan la importancia de los estudios multisesión ${ }^{30}$.

Wilksch y Wade llevaron a cabo en 2008 el programa "Media Smart" en Australia del Sur, con 540 estudiantes divididos en grupo intervención (Alfabetización mediática) y grupo control. Participaron tanto chicos como chicas en grupos de 16 y 26 estudiantes 
con edad media de 13,62. El programa consta de 8 sesiones de 50 minutos; se imparten 2 lecciones a la semana. Los cuestionarios para medir la autoestima corporal y la influencia del ideal estético se pasaron en cuatro ocasiones: preprograma y posprograma (al mes), a los 6 meses de seguimiento y a los 30 meses. Tanto en los varones como en las mujeres asignados al azar al grupo de Alfabetización mediática, se encontraron mejoras estadísticamente significativas tanto en la imagen corporal como en la internalización del ideal estético tanto en el posprograma como en todo el seguimiento, aunque siendo mayor en las mujeres.

Raich et al. diseñaron en 2001 un programa preliminar psicoeducativo de prevención de TCA con los siguientes componentes: Nutrición (NUT) dirigida hacia la corrección de falsas creencias sobre la nutrición y hacia la provisión de conocimientos de alimentación equilibrada; Alfabetización mediática (ML) y crítica del Modelo Estético-Belleza (AMC). Se desarrollaron en 2 sesiones semanales cada una de 1,5 horas. Para evaluar la eficacia de los diferentes componentes del estudio, un grupo recibió toda la intervención (NUT + AMC + ML), mientras que el otro recibió sólo el segundo componente (AMC + ML). Ambos grupos también fueron comparados con un grupo control sin intervención.

Se seleccionaron 349 niñas de $2^{\circ}$ de ESO en la ciudad de Terrassa, Barcelona. La evaluación previa a la intervención fue completada por 323 participantes. Las niñas tenían una edad promedio de 13,11 años. 288 participaron en el seguimiento de los 6 meses después.

Se encontraron cambios significativamente mayores en los dos grupos de intervención en comparación con el grupo de control de CIMEC Total. Entre el programa completo y el programa parcial sólo se observó diferencias en el aumento de conocimientos sobre nutrición al añadir el componente $\mathrm{NUT}^{19}$.

Por otro lado, P. Espinoza et al. aplicaron en 2013 el programa cuasi-experimental AMM-EC a 443 adolescentes, niñas y niños, de 12 a 14 años en la ciudad de Terrassa, Barcelona. El programa consta de dos componentes: Alfabetización mediática (ML) y nutrición (NUT) en versión completa (ML + NUT) o versión parcial (ML). Se realizó seguimiento a los 7 y a los 30 meses. El 62,3 \% tuvo un seguimiento a los 30 meses. El componente NUT consiste en una sesión de $90 \mathrm{~min}$ y el componente ML consiste en dos sesiones de 90 min y dos sesiones de $60 \mathrm{~min}$ (3 sesiones semanales durante la hora de clase). Utilizaron el cuestionario QÜIC para evaluar la satisfacción corporal y los problemas de imagen corporal de manera similar que la escala BES usada en nuestra investigación. Las puntuaciones de satisfacción corporal para ML + NUT aumentaron a los 7 meses y disminuyeron a los 30 meses de seguimiento (tendencia cuadrática); el Grupo ML se mantuvo constante en el tiempo ${ }^{31}$.

Los resultados de los estudios actuales sugieren la importancia de las sesiones de refuerzo, para compensar el efecto constante de la presión media, y la evaluación de la eficacia a largo plazo de los programas.

En los programas preventivos se deberían incluir los referentes de los adolescentes: padres, profesores, entrenadores, grupos de iguales y médicos ${ }^{11}$.

Para futuras investigaciones, consideramos necesario aumentar el tamaño de la muestra, añadir un grupo control, realizar sesiones de refuerzo y un seguimiento a corto y largo plazo.

En la actualidad se plantean varios debates sobre la orientación futura que debería impulsar a los nuevos programas de prevención en este ámbito. Básicamente, estos debates están relacionados con la adaptación de los futuros programas a la población masculina, las características idóneas de la población diana en cuanto a edad y nivel de riesgo, los contenidos específicos que deberían tener, la aproximación teórica en la que deberían fundamentarse, su intensidad y duración y, finalmente, el debate sobre la necesidad de integración de los campos de la prevención de los TCA y de la obesidad.

\section{Conclusiones}

- La alta prevalencia de los TCA y el hecho de que la anorexia nerviosa es una de las patologías psiquiátricas más graves, más difíciles de tratar y con una elevada tasa de mortalidad, pone en evidencia la necesidad de intervenir, con la intención de prevenir la aparición de los TCA, generando programas de prevención primaria que favorezcan la reducción de la vulnerabilidad de la población, ante los posibles factores de riesgo.

- No se han podido valorar las diferencias en el ideal estético y la autoestima en función de la edad, debido a que, de 10 sujetos, 8 tienen 15 años y 2 sujetos tienen 14 años.

- No se ha podido valorar la correlación entre la 
influencia del ideal estético y la autoestima con la repetición de curso, ya que sólo 1 de los 10 sujetos era repetidora.

- Se observa un efecto positivo de la intervención sobre la influencia del ideal estético estadísticamente significativo.

- Se observa un efecto positivo de la intervención en la autoestima corporal estadísticamente significativo.

- Se considera muy importante la labor de prevención por parte de la enfermera de salud mental y por eso se ha llevado a cabo este estudio de investigación con el objetivo de demostrar que nuestra intervención puede tener efectos positivos para reducir la vulnerabilidad de la población ante los posibles factores de riesgo.

\section{Referencias bibliográficas}

1. Peplau H. Relaciones Interpersonales en Enfermería. Un marco de referencia conceptual para la enfermería psicodinámica. BarceIona: Salvat; 1990. 6-35p.

2. Mejía M. Reflexiones sobre la relación interpersonal enfermerapaciente en el ámbito del cuidado clínico. Index Enfermermería[on line]. 2006;15(1699-5988):48-52.

3. Marriner, A y Raile M. Modelos y Teorías en Enfermería. 5a. Madrid: Elsevier Inc.; 2003. 379-385 p.

4. Wick K, Brix C, Bormann B, Sowa M, Strauss B, Berger U. Real-world effectiveness of a German school-based intervention for primary prevention of anorexia nervosa in preadolescent girls. Prev Med (Baltim) [Internet]. Elsevier Inc.; 2011;52(2):152-8. Available from: http://dx.doi.org/10.1016/j.ypmed.2010.11.022

5. Ruiz LPGZ-P de la UM de I del HCU de Z y CN de la J de A. Prevención de los trastornos de la conducta alimentaria. 1999;

6. Asociación Americana de Psiquiatría. Guía de consulta de los criterios diagnósticos del DSM 5. Arlington; 2013. 189-197 p.

7. Thompson C, Russell-mayhew S, Saraceni R. Eating Disorders : The Journal of Treatment \& Prevention Evaluating the Effects of a Peer-Support Model: Reducing Negative Body Esteem and Disordered Eating Attitudes and Behaviours in Grade Eight Girls Evaluating the Effects of a Peer-Support Model: Reducing Negative Body Esteem and Disordered Eating Attitudes and Behaviours in Grade Eight Girls. (October 2014):37-41.

8. House S, Loud K, Shubkin C. Female athlete triad for the primary care pediatrician. 2013;755-61.

9. Sundgot-borgen J, Meyer NL, Lohman TG, Ackland TR, Maughan RJ, Stewart $A D$, et al. How to minimise the health risks to athletes who compete in weight-sensitive sports review and position statement on behalf of the Ad Hoc Research Working Group on Body Composition, Health and Performance, under the auspices of the IOC Medical Commission. 2013;1012-22.

10. Neumark-sztainer D. School-Based Programs for Preventing Eating Disturbances. 1996;66(2):64-71.

11. Steiner-adair C, Sjostrom L, Franko DL, Pai S, Tucker R, Becker AE, et al. Primary Prevention of Risk Factors for Eating Disorders in Adolescent Girls: Learning From Practice. 2002;

12. Forman SF. Trastornos de la alimentación: Visión general del tratamiento [Internet]. UpToDate[Internet]citado el día 23/05/2017. 2017. Available from: http://uptodates.mhupa.csinet.es/contents/eating-disorders-overview-oftreatment?source=search_result\&search=prevenci\%C3\%B3n trastornos de la conducta alimentaria en adolescentes\&selectedTitle=3 150
13. Linville D, Cobb E, Lenee-bluhm T, Gabriela L, Gau JM, Stice E. Behaviour Research and Therapy Effectiveness of an eating disorder preventative intervention in primary care medical settings. 2015;75:32-9.

14. Sharpe H, Schober I, Treasure J, Schmidt U. Feasibility , acceptability and efficacy of a school-based prevention programme for eating disorders : cluster randomised controlled trial. 2013;428-35.

15. Yager Z, Diedrichs PC, Ricciardelli LA, Halliwell E. What works in secondary schools? A systematic review of classroom-based body image programs. Body Image [Internet]. Elsevier Ltd; 2013; Available from: http://dx.doi.org/10.1016/j.bodyim.2013.04.001

16. López-guimerà G, Sánchez-carracedo D, Fauquet J, Portell M, Raich RM. Impact of a School-Based Disordered Eating Prevention Program in Adolescent Girls : General and Specific Effects Depending on Adherence to the Interactive Activities. 2011;14(1):293-303.

17. Heather S et all. Preventing Eating Disorders. 2010;18(1):199-207.

18. Stice E, Shaw H. Eating Disorder Prevention Programs : A MetaAnalytic Review. 2004;130(2):206-27.

19. Raich RM, Sánchez-carracedo D, López- G, Portell M, Moncada A, Fauquet J. Eating Disorders : The Journal of Treatment \& Prevention A Controlled Assessment of School-Based Preventive Programs for Reducing Eating Disorder Risk Factors in Adolescent Spanish Girls. 2008; (January 2015):37-41.

20. Gaete P et all. Trastornos de la Conducta Alimentaria en Adolescentes y Jóvenes. 2012;23(5):566-78.

21. Rosen D. Identification and Management of Eating Disorders in Children and Adolescents abstract. Pediatrics. 2010;126:1240-53.

22. Negredo L. Factores de riesgo de la conducta suicida en internos con trastorno mental grave. 2010.

23. Sandoiu A. Anorexia: Deep brain stimulation may be an effective treatment. MedicalNewsToday[on line]. 2017;

24. Reyes-rodríguez L. Animando a su Hijo que Busque Ayuda. 2016;

25. Neumark-sztainer D, Levine MP, Paxton SJ, Smolak L, Piran N, Wertheim EH. Prevention of Body Dissatisfaction and Disordered Eating :What Next ?2006;265-85.

26. Manuel J, Alvarez G, Mancilla JM. Consistencia interna y estructura factorial del Cuestionario de Influencia de los Modelos Estéticos Corporales (CIMEC), en población mexicana. 2000;

27. Consumo MDESY. Guía de Práctica Clínica sobre Trastornos de la Conducta Alimentaria Guía de Práctica Clínica sobre Trastornos dela.

28. Jorquera M, Baños RM, Perpiñá $C$, Botella C. LA ESCALA DE ESTIMA CORPORAL ( BES ): VALIDACIÓN EN UNA MUESTRA ESPAÑOLA. 2005;173-92.

29. Gracia M De, Marcó M. Factores asociados a la conducta alimentaria en preadolescentes. 2007;19:646-53. 